Ponce SolÉ, Juli; Migliari, Wellington; Capdeferro VilLagrasa, Oscar (coords.): El derecho, la ciudad y la vivienda en la nueva concepción del desarrollo urbano: desafíos transnacionales y transdisciplinarios de la gobernanza en la Nueva Agenda Urbana

\title{
Ponce Solé, Juli; MigliarI, Wellington; Capdeferro Villagrasa, Oscar (coords.): El derecho, la ciudad y la vivienda en la nueva concepción del desarrollo urbano: desafíos transnacionales y transdisciplinarios de la gobernanza en la Nueva Agenda Urbana
}

Noel Armas Castilla Universidad de Sevilla (España) noel.armas@outlook.es

\section{NOTA BIOGRÁFICA}

Doble máster en Abogacía y Derecho Público por la Universidad de Sevilla. Doctorando por la misma institución. Personal Investigador en el marco del Programa de Empleo Joven de la Universidad de Sevilla en líneas relacionadas con las materias de Participación Ciudadana, Transparencia y Protección de Datos.

\section{RESUMEN}

Recensión de El derecho, la ciudad y la vivienda en la nueva concepción del desarrollo urbano: desafíos transnacionales y transdisciplinarios de la gobernanza en la Nueva Agenda Urbana, Juli Ponce SolÉ, Wellington Migliari, Oscar CAPDEFERRO VILLAGRASA (coords.). Editorial Atelier, Barcelona, 307 págs.

\section{PALABRAS CLAVE}

Nueva Agenda Urbana; Hábitat III.

\begin{abstract}
Review of El derecho, la ciudad y la vivienda en la nueva concepción del desarrollo urbano: desafíos transnacionales y transdisciplinarios de la gobernanza en la Nueva Agenda Urbana, Juli Ponce Solé, Wellington Migliari, Oscar Capdeferro Villagrasa (coords.). Editorial Atelier, Barcelona, 307 págs.
\end{abstract}

\section{KEYWORDS}

New Urban Agenda; Habitat III.

\section{INTRODUCCIÓN}

La aplicación de los objetivos comprometidos en la Nueva Agenda Urbana merece la observación de distintas materias interesantes para el Derecho Administrativo como pueden ser el Derecho a la Ciudad y, en general, las distintas formas de gestión pública orientadas a mejorar la calidad de vida en las ciudades desde la participación ciudadana o el empleo de las tecnologías en la ejecución de las políticas públicas locales. 
REALA. Nueva Época - N. ${ }^{13}$, abril-septiembre 2020 - ISSN: 1989-8975 - DOI: 10.24965/reala.i13.10748 - [Págs. 152-156]

PONCE SOlÉ, Juli; MigliarI, Wellington; CAPDEFERRo VilLAGRASA, Óscar (coords.): El derecho, la ciudad y la vivienda en la nueva ...

Noel Armas Castilla

Este interés se ve reforzado con el auge que están cobrando estas materias a la luz de diversas publicaciones como Droit de la Ville, en la que J. B. Auby ha sido pionero en el estudio del Derecho a la Ciudad, o la obra colectiva El derecho a la ciudad: el reto de las smart cities, dirigida por Vicenç Aguado i Cudolà, Vera Parisio, Òscar Casanovas i lbàñez (con sendas recensiones de mi autoría en la Revista Andaluza de la Administración Pública, núms. 103 y 104, respectivamente). Precisamente, estas cuestiones serán también estudiadas en el Congreso de la Asociación Española de Profesores de Derecho Administrativo que se celebrará en Ibiza en febrero de 2020, lo que acredita su actualidad.

El derecho, la ciudad y la vivienda en la nueva concepción del desarrollo urbano: desafíos transnacionales y transdisciplinarios de la gobernanza en la Nueva Agenda Urbana pretende ofrecer un acercamiento a las cuestiones jurídicas que plantea la ejecución de la Nueva Agenda Urbana. Los trabajos que componen el libro parten a veces de las experiencias en entidades locales concretas, y otras de posiciones eminentemente teórico-filosóficas, comparando doctrinas de distintos autores o profundizando en elementos específicos de la ciudad inteligente de interés para el ámbito del Derecho administrativo como es el catálogo de derechos conexos a este modelo de desarrollo urbano. Además, diversos trabajos insisten en la idea de que la aplicación de las tecnologías de la información con el uso de big data, las nuevas formas de gestión pública y de gobernanza es un pilar esencial en la construcción de la ciudad inteligente.

Así pues, conviene atender al contenido de la obra de manera pormenorizada y de acuerdo con las propias partes en que se estructura: una primera parte denominada "El derecho a la ciudad y la Nueva Agenda Urbana", en la que se focaliza el derecho a la ciudad en el contexto urbano; y una segunda parte, titulada "El derecho a la Ciudad, Gobernanza y la Nueva Agenda Urbana", que se nuclea alrededor de las distintas fórmulas de gobernanza disponibles para las autoridades. Hay que tener en cuenta que los objetivos de esta Nueva Agenda Urbana se refieren a los acuerdos alcanzados en la Tercera Conferencia de la ONU sobre Vivienda y Desarrollo Urbano Sostenible de 2016 (conocida como Hábitat III) y que renovó, no sin críticas, algunas de las metas globales a la luz del desarrollo de los anteriores objetivos y el estado actual de habitabilidad en las ciudades.

De esta manera, procedemos a recensionar ordenadamente los trabajos que componen el libro:

\section{PARTE I: EL DERECHO A LA CIUDAD Y LA NUEVA AGENDA URBANA}

"El usuario, titular del derecho a la ciudad", de Camille MIALOT. La autora analiza las políticas de participación que tienen en cuenta el papel del usuario frente al del residente en los procesos de decisión en materia de servicios públicos. El planteamiento consiste en que el usuario, en tanto que es quien hace uso de estos servicios, sea tenido en cuenta en los procesos de participación, por lo que personas como los turistas (que no encajan en la definición de habitante o ciudadano) puedan aportar una visión más realista de las necesidades de mejora de los servicios. Ello, a la postre, también genera la pregunta de saber en qué medida estos agentes tienen un derecho a la ciudad equiparable al de los ciudadanos tradicionales, con quienes pueden ocasionarse naturalmente fricciones. En todo caso, esta perspectiva del usuario como protagonista en las técnicas de participación refleja, a mi juicio, un enfoque muy interesante en el concepto que se tiene del propio instituto de la participación, al revisar un elemento de su configuración tan aparentemente aceptado como el de sus sujetos activos.

"La regularización del suelo urbano como instrumento de efectividad de las políticas públicas de protección a la población en situación de calle o personas sin hogar", de Ana Paula SANTOS DINIZ. La investigadora hace hincapié en el problema de exclusión social que padecen las personas sin hogar de los espacios urbanos, acentuado en las últimas décadas con motivo del crecimiento desordenado y desigual que han experimentado muchas ciudades del mundo. Considera que, en el marco de la Nueva Agenda Urbana, se pueden ejecutar instrumentos tendentes a revertir la situación de estas personas. La autora estudia el caso brasileño, donde se ha previsto la ejecución de instrumentos como la usucapión especial urbana, la Concesión de Derecho Real de Uso (CDRU) o la Concesión de uso especial para los fines de Vivienda (CUEM). Considero que puede ser interesante el estudio de estos instrumentos para su adaptación a los problemas de exclusión social en nuestras ciudades, atendiendo a la competencia en viviendas que las Entidades Locales tienen reconocidas en nuestro ordenamiento. Ello permitiría a estos sujetos la implantación de estas políticas en sus espacios de actuación.

"La judicialización del derecho a la ciudad como estrategia de la movilización judicial para la participación ciudadana", de Flavia Danielle SANTIAGO LIMA. La autora aborda el concepto de legal mobilization, al 
REALA. Nueva Época - N. ${ }^{13}$, abril-septiembre 2020 - ISSN: 1989-8975 - DOI: 10.24965/reala.i13.10748 - [Págs. 152-156]

PONCE SOlÉ, Juli; MigliarI, Wellington; CAPDEFERRo VilLAGRASA, Óscar (coords.): El derecho, la ciudad y la vivienda en la nueva ...

Noel Armas Castilla

que considera un instrumento útil para la consecución (u obstrucción) de determinadas políticas públicas a través del uso de acciones legales. Se estudian causas, consecuencias y eficacia de este instrumento como motor transformador de políticas públicas en el ámbito del derecho a la ciudad, y además se analiza el comportamiento de otros instrumentos y agentes paralelos a la judicialización de las políticas públicas. El trabajo expone el caso brasileño, desgranando el recorrido de los últimos años en los procesos judiciales vinculados a la política en el país sudamericano.

"Propuestas para un diálogo integrado e integrador: la nueva agenda urbana a la luz de Lefebvre, Joseph Comblin y Laudatio Si", de Marta LORA-TAMAYO VALLVÉ. La autora lleva a cabo un análisis sobre el derecho a la ciudad recogido en la Nueva Agenda Urbana desde una vertiente filosófica comparando posiciones de distintos teólogos como Lefebvre, el Papa Francisco I y Joseph Comblin, doctrinas en las que la autora selecciona algunos de sus objetos para la búsqueda de la verdad en un nuevo humanismo. De esta manera, la autora estudia la protección de la ciudad como bien en la observación de la ecología integral, el desarrollo sostenible, el destino de los bienes al bien común, la búsqueda de la prosperidad y la belleza, y la erradicación de la pobreza. A mi juicio, de este trabajo se desprende una interesante comparativa de doctrinas que, una vez estudiadas y contrastadas, ofrecen una visión filosófica amplia e integrada para la consecución de los objetivos de mejora de la vida humana en las ciudades.

\section{PARTE II: EL DERECHO A LA CIUDAD, GOBERNANZA Y LA NUEVA AGENDA URBANA}

"El derecho a la ciudad y la Nueva Agenda Urbana: una aproximación integrada y sistémica a los derechos en la ciudad, con referencia al derecho a la vivienda", de Juli PONCE SolÉ. Se hace un estudio sobre el concepto de derecho a la ciudad, así como de algunos de los elementos que lo integran como son sus pilares, componentes, protección jurídica, responsabilidades y protagonistas desde una perspectiva de derecho urbanístico. Se estructura el análisis del derecho a la ciudad a partir de su interacción con otros derechos, presentes en la ciudad, y que pueden converger con este derecho a la ciudad, a saber: el derecho a un desarrollo sostenible y al medio ambiente, el derecho a la igualdad y no discriminación, derecho a la vivienda y medio ambiente urbano, derecho a la educación no segregada, derecho a la libertad religiosa, a la seguridad urbana, al bueno gobierno y buena administración en el espacio urbano. En adición se realiza una mención a técnicas más punteras de gestión pública como son el establecimiento progresivo de las ciudades inteligentes y su significación para el alcance de ciudades más participativas y con mayor calidad de vida. Comporta especial interés no sólo el análisis de la materia sino su interacción con otros derechos que, relacionados, completan el catálogo de derechos que cabe esperar en el funcionamiento óptimo de una ciudad inteligente.

"La Gobernanza metropolitana y la nueva agenda urbana", de Mariona TomÀs. Estudia algunos de los caracteres de la gobernanza en áreas de aglomeraciones urbanas, al que considera relegado a un segundo plano de acuerdo con los tratamientos recibidos en las legislaciones de los países de nuestro entorno e incluso por la falta de formulación de un derecho a la ciudad en estos entornos en el marco de la Nueva Agenda Urbana. Elabora una exposición de distintos modelos de gobernanza metropolitana, de acuerdo con el carácter decreciente de su grado de institucionalización, entre los que establece y estudia los gobiernos metropolitanos, las agencias metropolitanas, la coordinación vertical y la cooperación voluntaria entre municipios, de los que además analiza sus variables de desarrollo a la luz de sus competencias, su financiación, su grado de representación democrática y participación ciudadana y sus relaciones multinivel en sentido vertical y horizontal.

Además, repasa y valora los avances normativos en el ámbito de la Unión Europea y de algunos de sus Estados Miembros, aunque considera necesario reivindicar un mayor protagonismo de las autoridades de las áreas metropolitanas y una mejora de recursos, atendiendo al hecho de que las aglomeraciones urbanas albergan a un segmento mayoritario de la población mundial, una importante cifra del volumen de economía generada en espacios como el europeo, su papel en la respuesta a determinados retos globales como el cambio climático y las desigualdades sociales, entre otras.

"Dignidad humana, esfera pública y nuevos retos a los gobiernos locales", de Wellington MIGLIARI. EI investigador comienza su trabajo recordando los estadios por los que históricamente han pasado los usos de los espacios urbanos. El núcleo de este trabajo residen en la dignidad humana con base en fórmulas de participación, y cuya relación con la Nueva Agenda Urbana es evidente por cuanto se formulan los instrumentos en los que pueden reposar algunas de las metas propias del derecho a la ciudad como es el aumento de los índices de inclusión en la vida urbana, pero también todo un catálogo de derechos como el de acceso a 
REALA. Nueva Época - N. ${ }^{13}$, abril-septiembre 2020 - ISSN: 1989-8975 - DOI: 10.24965/reala.i13.10748 - [Págs. 152-156]

PONCE SOlÉ, Juli; MigliarI, Wellington; CAPDEFERRo VilLAGRASA, Óscar (coords.): El derecho, la ciudad y la vivienda en la nueva ...

Noel Armas Castilla

la información pública y la transparencia, el acceso y disfrute del medio ambiente en un desarrollo humano sostenible en el que se contemple la balanza de intereses a veces contrapuesto entre particulares y la sociedad, y que encuentra un buen nicho en espacios donde se observa y potencia la incorporación de iniciativas como la permacultura (es decir el diseño de hábitats humanos sostenibles), entre otras políticas públicas al abrigo de la Nueva Agenda Urbana.

"Urbanismo y corrupción en la era de la inteligencia artificial", de Oscar CAPDEFERRo ViLLAGRASA. Los avances realizados en la actualización de objetivos y compromisos de la Nueva Agenda Urbana, cuyo estudio se centra en los altos índices y costos de la corrupción en el desarrollo igualitario, integrado y sostenible de determinadas ciudades. Además, se señala la posibilidad que brindan algunos mecanismos dotados de inteligencia artificial para prever y gestionar riesgos de corrupción, destacando los modelos más comunes de gestión de riesgo como el operational risk management, en las tareas de control de la administración española. Según el autor, la utilización de estas herramientas ha de servir para la mejora y optimización de los mecanismos de control y gestión de riesgos del sector público, especialmente si los agentes sociopolíticos responsables desean avanzar en la consecución de objetivos propuestos por la Nueva Agenda Urbana, con la integración de las mejoras técnicas en el desarrollo de la actividad de la Administración inteligente e informatizada. Estas herramientas son de interés no sólo para la consecución de tales objetivos sino para la optimización de la lucha contra la corrupción en nuestras Administraciones con el empleo de mecanismos cada vez más eficaces y modernos.

"Datos y algoritmos en la ciudad inteligente", de Clara I. VELASCO RICO. El objeto de este trabajo aborda las posibilidades de uso que presentan los software y algoritmos en el funcionamiento y desarrollo de la ciudad inteligente. Expone que las herramientas de inteligencia artificial en las que se instrumentaliza la gobernanza algorítmica suponen un reto para su uso por parte de las entidades locales. De acuerdo con la autora, este flujo de información ha de confrontarse con la normativa sobre protección de datos acceso y reutilización de la información, planteándose importantes conflictos jurídicos.

"Crisis, juicios políticos y judicialización de la gobernabilidad: el STF y la estabilización del sistema político", de Alexandre Douglas ZAIDAN DE CARVALHO. El autor analiza el caso brasileño, y más concretamente, las cuestiones relacionadas con el juicio al que fue sometido su expresidenta Dilma Rousseff y las consecuencias para la gobernabilidad del país con motivo de la participación del poder judicial en la resolución de fricciones entre los poderes ejecutivo y legislativo. Al Tribunal Supremo brasileño se le estaría encomendando con este fenómeno de judicialización el arbitraje entre la Cámara legislativa y el Gobierno en una crisis marcada por la fuerte polarización del electorado, el debilitamiento de los partidos políticos, subida de los niveles de conflicto social y falta de confianza en los agentes políticos para la lucha contra la corrupción. Es en estas circunstancias que, a la postre, el poder judicial debe ser capaz de asumir un papel de regeneración y recuperación de la opinión pública, mediante la depuración de las responsabilidades originadas por los integrantes de los poderes legislativo y ejecutivo, la administración y salvaguarda de la gobernabilidad del Estado, fuertemente comprometida como resultado de la crisis política en Brasil.

"Planificación urbana, salud colectiva y ciudades saludables: propuestas y contradicciones a partir de un análisis histórico y macrofilosófico", de André Luiz FREITAS Dias y Gonçal MAYOS SOLSONA. Los autores consideran que, desde el surgimiento de la vida urbana a partir de la Edad Media en Europa, la insalubridad ha sido un problema constante para la convivencia y desarrollo de estos entornos, lo que pasa a tener mayor entidad en nuestros días al estimarse que más de la mitad de la población mundial vive en ciudades. Se realiza un repaso histórico de los estadios alcanzados en la mejora de la salubridad especialmente en lo que se refiere a los inicios de la planificación urbanística y saneamiento urbanos, la higiene y la vivienda y las nuevas perspectivas de salud que diferentes actores político-sociales reivindican y que en algunos casos ha llegado a vertebrarse a través de organizaciones supranacionales como el Movimiento Internacional de Ciudades y Municipios Saludables, que vienen a poner de manifiesto el interés por la mejora de la calidad de vida y la concreción de medidas, instrumentos y derechos en los espacios urbanos como una preocupación compartida por el problema global de la habitabilidad y calidad de vida en la ciudad.

La obra recensionada conforma, en definitiva, una recopilación de trabajos que giran alrededor de la noción de derecho a la ciudad, en los que se plantean ejemplos de distintas ciudades sudamericanas y europeas, aportaciones que, por su variedad, permiten enriquecer la visión del fenómeno y no impiden alcanzar conclusiones compartibles, puesto que determinados problemas presentes en las ciudades, como la desigualdad social o la contaminación, son comunes y no entienden de fronteras. Es por este motivo que los ejemplos propuestos contemplan no sólo el estudio de jurisprudencia, normas y planes nacionales, regiona- 
REALA. Nueva Época - N. 13, abril-septiembre 2020 - ISSN: 1989-8975 - DOI: 10.24965/reala.i13.10748 - [Págs. 152-156]

Ponce SolÉ, Juli; MigliarI, Wellington; CAPDEFERRo Villagrasa, Óscar (coords.): El derecho, la ciudad y la vivienda en la nueva ...

Noel Armas Castilla

les o locales, sino también las directrices marcadas por la Nueva Agenda Urbana. En efecto, este documento es mencionado en múltiples ocasiones a lo largo de la obra, como instrumento de gran relevancia para la búsqueda colectiva a soluciones jurídicas adaptables a la realidad de las ciudades en el mundo. Con él, en fin, la ONU evidencia la necesidad de diseñar y compartir programas de participación que ayuden a las Administraciones locales y regionales de todo el mundo a mitigar los problemas propios de sus espacios urbanos con la aplicación de técnicas de gobernanza más eficientes, modernas y sostenibles. 